\title{
ON SUCCESSIVE RADII OF $p$-SUMS OF CONVEX BODIES
}

\author{
BERNARDO GONZÁLEZ AND MARÍA A. HERNÁNDEZ CIFRE
}

\begin{abstract}
We study the behavior of the so called successive inner and outer radii with respect to the $p$-sums of convex bodies, $p \geq 1$, which were introduced by Firey in 1962 . For all $p \geq 1$ we get the upper and lower bounds for the radii of the $p$-sum of two convex bodies in terms of the radii of the sets. These results generalize the corresponding relations for the classical Minkowski addition.
\end{abstract}

\section{INTRODUCTION}

Let $\mathcal{K}^{n}$ be the set of all convex bodies, i.e., compact convex sets, in the $n$-dimensional Euclidean space $\mathbb{R}^{n}$. Let $\langle\cdot, \cdot\rangle$ and $|\cdot|_{2}$ be the standard inner product and the Euclidean norm in $\mathbb{R}^{n}$, respectively, and denote by $\mathrm{e}_{i}$ the $i$-th canonical unit vector.

The set of all $i$-dimensional linear subspaces of $\mathbb{R}^{n}$ is denoted by $\mathcal{L}_{i}^{n}$, and for $L \in \mathcal{L}_{i}^{n}, L^{\perp}$ denotes its orthogonal complement. For $K \in \mathcal{K}^{n}$ and $L \in \mathcal{L}_{i}^{n}$, the orthogonal projection of $K$ onto $L$ is denoted by $K \mid L$. With $\operatorname{lin}\left\{u_{1}, \ldots, u_{m}\right\}$ we represent the linear hull of the vectors $u_{1}, \ldots, u_{m}$ and with $\left[u_{1}, u_{2}\right]$ the line segment with end-points $u_{1}, u_{2}$. Finally, for $S \subset \mathbb{R}^{n}$ we denote by conv $S$ the convex hull of $S$ and by bd $S$ its boundary. Moreover, we write relbd $S$ to denote the relative boundary of $S$, i.e., the boundary of $S$ relative to its affine hull aff $S$.

The diameter, minimal width, circumradius and inradius of a convex body $K$ are denoted by $\mathrm{D}(K), \omega(K), \mathrm{R}(K)$ and $\mathrm{r}(K)$, respectively. For more information on these functionals and their properties we refer to [4, pp. 5659]. If $f$ is a functional on $\mathcal{K}^{n}$ depending on the dimension in which a convex body $K$ is embedded, and if $K$ is contained in an affine space $A$, then we write $f(K ; A)$ to stress that $f$ has to be evaluated with respect to the space $A$. Successive outer and inner radii are defined in the following way.

Definition 1.1. For $K \in \mathcal{K}^{n}$ and $i=1, \ldots, n$ let

$$
\mathrm{R}_{i}(K)=\min _{L \in \mathcal{L}_{i}^{n}} \mathrm{R}(K \mid L) \quad \text { and } \quad \mathrm{r}_{i}(K)=\max _{L \in \mathcal{L}_{i}^{n}} \max _{x \in L^{\perp}} \mathrm{r}(K \cap(x+L) ; x+L) .
$$

2000 Mathematics Subject Classification. Primary 52A20; Secondary 52A40.

Key words and phrases. Successive inner and outer radii, $p$-sum.

Authors are supported by MCI-FEDER, MTM2009-10418, and by "Programa de Ayudas a Grupos de Excelencia de la Región de Murcia", Fundación Séneca (Plan Regional de Ciencia y Tecnología 2007/2010), 04540/GERM/06. 
Notice that $\mathrm{R}_{i}(K)$ is the smallest radius of a solid cylinder with $i$-dimensional spherical cross section containing $K$, and $\mathrm{r}_{i}(K)$ is the radius of the greatest $i$-dimensional ball contained in $K$. It is clear that the outer radii are increasing in $i$, whereas the inner radii are decreasing in $i$, and we obviously have

$$
\mathrm{R}_{n}(K)=\mathrm{R}(K), \mathrm{R}_{1}(K)=\frac{\omega(K)}{2}, \mathrm{r}_{n}(K)=\mathrm{r}(K) \text { and } \mathrm{r}_{1}(K)=\frac{\mathrm{D}(K)}{2} .
$$

The first systematic study of the successive radii was developed in [1. For more information on these radii and their relation with other measures, we refer, for instance, to [1, 2, 15, 8, 11, 13, 14, 15, 19, 20, 21, 22, 24].

From now on we write $\mathcal{K}_{0}^{n}$ to denote the class of convex bodies containing the origin. In [7] Firey introduced the following generalization of the classical Minkowski addition (i.e., vectorial addition). Let $p \geq 1$ be given. Then, for $K, K^{\prime} \in \mathcal{K}_{0}^{n}$, the $p$-sum of $K$ and $K^{\prime}$ is the unique convex body $K+{ }_{p} K^{\prime}$ for which the support function

$$
h\left(K+{ }_{p} K^{\prime}, \cdot\right)^{p}=h(K, \cdot)^{p}+h\left(K^{\prime}, \cdot\right)^{p} .
$$

We recall that $h(K, u)=\max \{\langle x, u\rangle: x \in K\}, u \in \mathbb{S}^{n-1}$; as usual, $\mathbb{S}^{n-1}$ denotes the $(n-1)$-dimensional unit sphere of $\mathbb{R}^{n}$ (see e.g. [23, s. 1.7]). Clearly, when $p=1$, formula (1) defines the usual Minkowski sum $K+K^{\prime}$, and for $p=\infty$ it holds $h\left(K+\infty K^{\prime}, u\right)=\max \left\{h(K, u), h\left(K^{\prime}, u\right)\right\}$, i.e., $K+_{\infty} K^{\prime}=\operatorname{conv}\left(K \cup K^{\prime}\right)$. Moreover, in [7, Theorem 1] it is shown that

$$
K+{ }_{q} K^{\prime} \subseteq K+{ }_{p} K^{\prime}
$$

for all $1 \leq p \leq q$. Observe that for the $p$-sums of sets, except in the case $p=1$, the translation invariance is lost.

In [16, 17 Lutwak studied $p$-sums of convex bodies systematically, and developed a theory nowadays known as Brunn-Minkowski-Firey theory. In the last years many important developments of this theory have come out; we mention e.g. [3, 6, 18, and the references inside.

In this point we would like to notice that usually $p$-sums are defined for convex bodies containing the origin as a relative interior point, since this condition is needed in some aspects of the Brunn-Minkowski-Firey theory; however, regarding the functionals we are working on, this condition can be withdrawn, and thus we allow the origin to lie on the boundary of the convex bodies.

The behavior of the diameter, minimal width, circumradius and inradius with respect to the Minkowski sum is well known (see e.g. [23, p. 42]), namely,

$$
\begin{array}{ll}
\mathrm{D}\left(K+K^{\prime}\right) \leq \mathrm{D}(K)+\mathrm{D}\left(K^{\prime}\right), & \omega\left(K+K^{\prime}\right) \geq \omega(K)+\omega\left(K^{\prime}\right), \\
\mathrm{R}\left(K+K^{\prime}\right) \leq \mathrm{R}(K)+\mathrm{R}\left(K^{\prime}\right), & \mathrm{r}\left(K+K^{\prime}\right) \geq \mathrm{r}(K)+\mathrm{r}\left(K^{\prime}\right),
\end{array}
$$

and in 9] we obtained the corresponding relations for the more general successive inner and outer radii. Here we are interested in generalizing those results to the $p$-sums of convex bodies. We prove the following theorems. 
Theorem 1.1. Let $K, K^{\prime} \in \mathcal{K}_{0}^{n}$ and $p \geq 1$. Then

$$
\begin{aligned}
2^{\frac{p-1}{p}} \mathrm{R}_{1}\left(K+{ }_{p} K^{\prime}\right) & \geq \mathrm{R}_{1}(K)+\mathrm{R}_{1}\left(K^{\prime}\right) \quad \text { for all } p \geq 1, \\
2^{\frac{3 p-2}{2 p}} \mathrm{R}_{i}\left(K+{ }_{p} K^{\prime}\right) & \geq \mathrm{R}_{i}(K)+\mathrm{R}_{i}\left(K^{\prime}\right) \quad \text { for } 1 \leq p \leq 2, i=2, \ldots, n, \\
\mathrm{R}_{i}\left(K+{ }_{p} K^{\prime}\right) & \geq \max \left\{\mathrm{R}_{i}(K), \mathrm{R}_{i}\left(K^{\prime}\right)\right\} \quad \text { for } p \geq 2, i=2, \ldots, n .
\end{aligned}
$$

All inequalities are best possible.

Moreover, a reverse inequality can be obtained only in the case of the circumradius:

Proposition 1.1. Let $K, K^{\prime} \in \mathcal{K}_{0}^{n}$ and $p \geq 1$. Then

$$
\mathrm{R}_{n}\left(K+{ }_{p} K^{\prime}\right) \leq \mathrm{R}_{n}(K)+\mathrm{R}_{n}\left(K^{\prime}\right),
$$

which is tight, and for any $i=1, \ldots, n-1$, there exists no constant $c>0$ such that $c \mathrm{R}_{i}\left(K+{ }_{p} K^{\prime}\right) \leq \mathrm{R}_{i}(K)+\mathrm{R}_{i}\left(K^{\prime}\right)$.

Theorem 1.1 and Proposition 1.1 become [9, Theorem 1.1 and Remark 3.1] when $p=1$.

Theorem 1.2. Let $K, K^{\prime} \in \mathcal{K}_{0}^{n}$ and $p \geq 1$. Then

$$
\begin{aligned}
2^{\frac{p-1}{p}} \mathrm{r}_{n}\left(K+{ }_{p} K^{\prime}\right) & \geq \mathrm{r}_{n}(K)+\mathrm{r}_{n}\left(K^{\prime}\right) \quad \text { for all } p \geq 1 \\
2^{\frac{3 p-2}{2 p}} \mathrm{r}_{i}\left(K+{ }_{p} K^{\prime}\right) & \geq \mathrm{r}_{i}(K)+\mathrm{r}_{i}\left(K^{\prime}\right) \quad \text { for } 1 \leq p \leq 2, i=1, \ldots, n-1, \\
\mathrm{r}_{i}\left(K+{ }_{p} K^{\prime}\right) & \geq \max \left\{\mathrm{r}_{i}(K), \mathrm{r}_{i}\left(K^{\prime}\right)\right\} \quad \text { for } p \geq 2, i=1, \ldots, n-1 .
\end{aligned}
$$

All inequalities are best possible.

A reverse inequality can be obtained only in the case of the diameter:

Proposition 1.2. Let $K, K^{\prime} \in \mathcal{K}_{0}^{n}$ and $p \geq 1$. Then

$$
\mathrm{r}_{1}\left(K+{ }_{p} K^{\prime}\right) \leq \mathrm{r}_{1}(K)+\mathrm{r}_{1}\left(K^{\prime}\right),
$$

which is tight, and for any $i=2, \ldots, n$, there exists no constant $c>0$ such that $c \mathrm{r}_{i}\left(K+{ }_{p} K^{\prime}\right) \leq \mathrm{r}_{i}(K)+\mathrm{r}_{i}\left(K^{\prime}\right)$.

Theorem 1.2 and Proposition 1.2 become [9, Theorem 1.2 and Remark 3.2] when $p=1$.

We notice that in both Theorems 1.1 and 1.2 , the last two inequalities are valid for all $p \geq 1$. We point out that the distinction depending on the range of $p$ is needed for the sharpness.

In Section 2 we present the proofs of the main results, as well as some remarks. Finally, Section 3 is devoted to study a particular case for which the bounds in Theorems 1.1 and 1.2 can be improved, the so called $p$-difference body of a convex set $K$, i.e., $K+{ }_{p}(-K)$. 


\section{Proofs of the main Results}

For $p \geq 1$ let $B_{n}^{p}$ be the unit $p$-ball associated to the $p$-norm $|\cdot|_{p}$, i.e.,

$$
B_{n}^{p}=\left\{x=\left(x_{1}, \ldots, x_{n}\right) \in \mathbb{R}^{n}:|x|_{p}=\left(\sum_{i=1}^{n}\left|x_{i}\right|^{p}\right)^{1 / p} \leq 1\right\},
$$

with $|x|_{\infty}=\max \left\{\left|x_{i}\right|: i=1, \ldots, n\right\}$. For the sake of brevity, we will write $B_{n}=B_{n}^{2}$ to denote the $n$-dimensional Euclidean unit ball. Moreover, for $L \in \mathcal{L}_{i}^{n}$, we will write $B_{i, L}=B_{n} \cap L$ and $B_{i, L}^{p}=B_{n}^{p} \cap L$.

On the one hand, for any $1 \leq p \leq q$, it is a direct consequence of Hölder's inequality for $q / p$ (see e.g. [12, p. 15]) that $|\cdot|_{q} \leq|\cdot|_{p} \leq n^{1 / p-1 / q}|\cdot|_{q}$, which is equivalent to the inclusions $B_{n}^{p} \subseteq B_{n}^{q} \subseteq n^{1 / p-1 / q} B_{n}^{p}$. On the other hand, it is known (see e.g. [10]) that $\left[-\mathrm{e}_{1}, \mathrm{e}_{1}\right]+{ }_{p} \cdots+{ }_{p}\left[-\mathrm{e}_{n}, \mathrm{e}_{n}\right]=B_{n}^{q}$ for $q \geq 1$ such that $1 / p+1 / q=1$. Therefore we get, in particular, that

$$
\left[-\mathrm{e}_{1}, \mathrm{e}_{1}\right]+{ }_{p} \cdots+{ }_{p}\left[-\mathrm{e}_{n}, \mathrm{e}_{n}\right] \subseteq \begin{cases}n^{1 / p-1 / 2} B_{n} & \text { for } 1 \leq p \leq 2, \\ B_{n} & \text { for } p \geq 2\end{cases}
$$

We start proving the lower bound for the outer radii $\mathrm{R}_{i}\left(K+{ }_{p} K^{\prime}\right)$ of the $p$-sum of two convex bodies in terms of the corresponding radii.

Proof of Theorem 1.1. In [7, Theorem 1] it is shown that

$$
\frac{1}{2^{(p-1) / p}}\left(K+K^{\prime}\right) \subseteq K+{ }_{p} K^{\prime} \subseteq K+K^{\prime}
$$

Therefore $2^{(p-1) / p} \mathrm{R}_{i}\left(K+{ }_{p} K^{\prime}\right) \geq \mathrm{R}_{i}\left(K+K^{\prime}\right)$ for all $i=1, \ldots, n$, and by 9 , Theorem 1.1] we get

$$
\begin{aligned}
& 2^{(p-1) / p} \mathrm{R}_{1}\left(K+{ }_{p} K^{\prime}\right) \geq \mathrm{R}_{1}\left(K+K^{\prime}\right) \geq \mathrm{R}_{1}(K)+\mathrm{R}_{1}\left(K^{\prime}\right), \\
& 2^{(p-1) / p} \mathrm{R}_{i}\left(K+{ }_{p} K^{\prime}\right) \geq \mathrm{R}_{i}\left(K+K^{\prime}\right) \geq \frac{1}{\sqrt{2}}\left(\mathrm{R}_{i}(K)+\mathrm{R}_{i}\left(K^{\prime}\right)\right),
\end{aligned}
$$

$i=2, \ldots, n$, which shows inequalities (4) and (5). Notice also that it always holds $K, K^{\prime} \subseteq K+{ }_{p} K^{\prime}$, which leads to

$$
\mathrm{R}_{i}\left(K+_{p} K^{\prime}\right) \geq \max \left\{\mathrm{R}_{i}(K), \mathrm{R}_{i}\left(K^{\prime}\right)\right\} .
$$

Since for any non-negative real numbers $a, b \geq 0$ it holds that if $p \geq 2$ then $\max \{a, b\} \geq 1 / 2^{(3 p-2) /(2 p)}(a+b)$, inequality $(6)$ is obtained.

So it remains to be shown that the three inequalities are best possible. For the first one, let $K=K^{\prime}$. Then $K+{ }_{p} K=2^{1 / p} K$ and thus

$$
2^{(p-1) / p} \mathrm{R}_{1}\left(K+{ }_{p} K\right)=2^{(p-1) / p} 2^{1 / p} \mathrm{R}_{1}(K)=\mathrm{R}_{1}(K)+\mathrm{R}_{1}(K) .
$$

Next, for $i \in\{2, \ldots, n-1\}$ we consider the convex bodies

$$
K=\left[-\mathrm{e}_{1}, \mathrm{e}_{1}\right]+\sum_{k=i+1}^{n}\left[-\mathrm{e}_{k}, \mathrm{e}_{k}\right], \quad K^{\prime}=\left[-\mathrm{e}_{2}, \mathrm{e}_{2}\right]+\sum_{k=i+1}^{n}\left[-\mathrm{e}_{k}, \mathrm{e}_{k}\right],
$$


i.e., the 0 -symmetric $(n-i+1)$-cubes with edges parallel to the coordinate axes and length 2 , of the subspaces $L_{j}=\operatorname{lin}\left\{\mathrm{e}_{j}, \mathrm{e}_{i+1}, \ldots, \mathrm{e}_{n}\right\} \in \mathcal{L}_{n-i+1}^{n}$, $j=1,2$, and for $i=n$ we take $K=\left[-\mathrm{e}_{1}, \mathrm{e}_{1}\right], K^{\prime}=\left[-\mathrm{e}_{2}, \mathrm{e}_{2}\right]$. Clearly $\mathrm{R}(K \mid L), \mathrm{R}\left(K^{\prime} \mid L\right) \geq 1$ for all $L \in \mathcal{L}_{i}^{n}$. Moreover, $\mathrm{R}\left(K \mid \operatorname{lin}\left\{\mathrm{e}_{1}, \ldots, \mathrm{e}_{i}\right\}\right)=$ $\mathrm{R}\left(K^{\prime} \mid \operatorname{lin}\left\{\mathrm{e}_{1}, \ldots, \mathrm{e}_{i}\right\}\right)=1$, which shows that $\mathrm{R}_{i}(K)=\mathrm{R}_{i}\left(K^{\prime}\right)=1$.

Let $1 \leq p \leq 2$, and we compute $\mathrm{R}_{i}\left(K+{ }_{p} K^{\prime}\right)$. Let $L \in \mathcal{L}_{i}^{n}$. On the one hand, since $\operatorname{dim} L_{j} \cap L \geq(n-i+1)+i-n=1$, there exist $x \in K \cap L$ and $x^{\prime} \in K^{\prime} \cap L$ with $|x|_{2},\left|x^{\prime}\right|_{2} \geq 1$, and because of the central symmetry, we may assume that $\left\langle x, x^{\prime}\right\rangle \geq 0$. Then

$$
\left|\frac{\left(x+x^{\prime}\right)}{2^{(p-1) / p}}\right|_{2} \geq \frac{\left(|x|_{2}^{2}+\left|x^{\prime}\right|_{2}^{2}\right)^{1 / 2}}{2^{(p-1) / p}} \geq \frac{2^{1 / 2}}{2^{(p-1) / p}}=2^{(2-p) /(2 p)}
$$

and thus, since $\left(x+x^{\prime}\right) / 2^{(p-1) / p} \in\left(K+{ }_{p} K^{\prime}\right) \cap L$ (see (11)), we get

$$
\mathrm{R}\left(\left(K+{ }_{p} K^{\prime}\right) \mid L\right) \geq \mathrm{R}\left(\left(K+{ }_{p} K^{\prime}\right) \cap L\right) \geq 2^{(2-p) /(2 p)}
$$

for all $L \in \mathcal{L}_{i}^{n}$. On the other hand, since the orthogonal projection of the $p$-sum of two convex bodies onto any lower dimensional linear subspace is the $p$-sum of the projections (see [7, pp. 21-22]), and using (10), we get

$$
\begin{aligned}
\left(K+{ }_{p} K^{\prime}\right) \mid \operatorname{lin}\left\{\mathrm{e}_{1}, \ldots, \mathrm{e}_{i}\right\} & =K\left|\operatorname{lin}\left\{e_{1}, \ldots, e_{i}\right\}+{ }_{p} K^{\prime}\right| \operatorname{lin}\left\{e_{1}, \ldots, e_{i}\right\} \\
& =K\left|\operatorname{lin}\left\{\mathrm{e}_{1}\right\}+{ }_{p} K^{\prime}\right| \operatorname{lin}\left\{\mathrm{e}_{2}\right\}=\left[-\mathrm{e}_{1}, \mathrm{e}_{1}\right]+{ }_{p}\left[-\mathrm{e}_{2}, \mathrm{e}_{2}\right] \\
& \subseteq 2^{(2-p) /(2 p)} B_{2, \operatorname{lin}\left\{\mathrm{e}_{1}, \mathrm{e}_{2}\right\}},
\end{aligned}
$$

which gives $\mathrm{R}\left(\left(K+{ }_{p} K^{\prime}\right) \mid \operatorname{lin}\left\{e_{1}, \ldots, e_{i}\right\}\right) \leq 2^{(2-p) /(2 p)}$. Then, together with 12. we get $\mathrm{R}\left(\left(K+{ }_{p} K^{\prime}\right) \mid \operatorname{lin}\left\{e_{1}, \ldots, e_{i}\right\}\right)=2^{(2-p) /(2 p)}$, and moreover,

$$
\begin{aligned}
\mathrm{R}_{i}\left(K+{ }_{p} K^{\prime}\right) & =\min _{L \in \mathcal{L}_{i}^{n}} \mathrm{R}\left(\left(K+{ }_{p} K^{\prime}\right) \mid L\right)=2^{(2-p) /(2 p)}=\frac{1}{2^{(3 p-2) /(2 p)}}(1+1) \\
& =\frac{1}{2^{(3 p-2) /(2 p)}}\left(\mathrm{R}_{i}(K)+\mathrm{R}_{i}\left(K^{\prime}\right)\right),
\end{aligned}
$$

as required.

Now let $p \geq 2$ and $K, K^{\prime}$ as before. First notice that $\mathrm{R}_{i}\left(K+{ }_{p} K^{\prime}\right) \geq$ $\mathrm{R}_{i}(K)=\mathrm{R}_{i}\left(K^{\prime}\right)=1$. With an analogous argument to the previous one, but using (10) when $p \geq 2$, we get that

$$
\mathrm{R}\left(\left(K+{ }_{p} K^{\prime}\right) \mid \operatorname{lin}\left\{e_{1}, \ldots, e_{i}\right\}\right) \leq \mathrm{R}\left(B_{2, \operatorname{lin}\left\{\mathrm{e}_{1}, \mathrm{e}_{2}\right\}}\right)=1 .
$$

Both inequalities give $\mathrm{R}_{i}\left(K+{ }_{p} K^{\prime}\right)=1=\mathrm{R}_{i}(K)=\mathrm{R}_{i}\left(K^{\prime}\right)$.

However, as we show next, there is no chance to get a reverse inequality for all outer radii.

Proof of Proposition 1.1. In the case of the circumradius we easily get, using (11) and (3), that

$$
\mathrm{R}_{n}\left(K+{ }_{p} K^{\prime}\right) \leq \mathrm{R}_{n}\left(K+K^{\prime}\right) \leq \mathrm{R}_{n}(K)+\mathrm{R}_{n}\left(K^{\prime}\right) .
$$

Notice that the inequality is tight, because the circumradius is a continuous functional and equality is attained if $K^{\prime}=\{0\}$. 
In order to show the non-existence of a reverse inequality, $i=1, \ldots, n-1$, we take the convex bodies

$$
K=\left[-\mathrm{e}_{n-i+1}, \mathrm{e}_{n-i+1}\right] \quad \text { and } \quad K^{\prime}=\sum_{k=1}^{n-i}\left[-\mathrm{e}_{k}, \mathrm{e}_{k}\right] .
$$

On the one hand, since

$$
K\left|\operatorname{lin}\left\{\mathrm{e}_{n-i}, \mathrm{e}_{n-i+2}, \ldots, \mathrm{e}_{n}\right\}=K^{\prime}\right| \operatorname{lin}\left\{\mathrm{e}_{n-i+1}, \ldots, \mathrm{e}_{n}\right\}=\{0\},
$$

then $\mathrm{R}_{i}(K)=\mathrm{R}_{i}\left(K^{\prime}\right)=0$, i.e., $\mathrm{R}_{i}(K)+\mathrm{R}_{i}\left(K^{\prime}\right)=0$. On the other hand,

$$
K+{ }_{p} K^{\prime} \supseteq \frac{1}{2^{(p-1) / p}}\left(K+K^{\prime}\right)=\frac{1}{2^{(p-1) / p}} \sum_{j=1}^{n-i+1}\left[-\mathrm{e}_{j}, \mathrm{e}_{j}\right],
$$

i.e., $K+{ }_{p} K^{\prime}$ contains an $(n-i+1)$-dimensional convex body, which implies that $\operatorname{dim}\left(\left(K+{ }_{p} K^{\prime}\right) \mid L\right) \geq 1$ for all $L \in \mathcal{L}_{i}^{n}$. Then, $\mathrm{R}_{i}\left(K+{ }_{p} K^{\prime}\right)>0$, which shows the result.

Remark 2.1. We notice that the inequality $\mathrm{R}\left(K+{ }_{p} K^{\prime}\right) \leq \mathrm{R}(K)+\mathrm{R}\left(K^{\prime}\right)$, $p \geq 1$, can be strengthened in the particular case when the circumcenter of both $K, K^{\prime} \in \mathcal{K}_{0}^{n}$ lies at the origin: since

$$
h\left(K+{ }_{p} K^{\prime}, u\right)=\left(h(K, u)^{p}+h\left(K^{\prime}, u\right)^{p}\right)^{1 / p} \leq\left(\mathrm{R}(K)^{p}+\mathrm{R}\left(K^{\prime}\right)^{p}\right)^{1 / p}
$$

for all $u \in \mathbb{S}^{n-1}$, then we get

$$
\mathrm{R}\left(K+{ }_{p} K^{\prime}\right) \leq\left(\mathrm{R}(K)^{p}+\mathrm{R}\left(K^{\prime}\right)^{p}\right)^{1 / p} .
$$

If $K^{\prime}=K$ equality holds.

Now we deal with the inner radii $\mathrm{r}_{i}\left(K+{ }_{p} K^{\prime}\right)$ and prove Theorem 1.2 .

Proof of Theorem 1.2. By (11) we have $2^{(p-1) / p} \mathrm{r}_{i}\left(K+{ }_{p} K^{\prime}\right) \geq \mathrm{r}_{i}\left(K+K^{\prime}\right)$ for all $i=1, \ldots, n$, and applying [9, Theorem 1.2] we get

$$
\begin{aligned}
& 2^{(p-1) / p} \mathrm{r}_{n}\left(K+{ }_{p} K^{\prime}\right) \geq \mathrm{r}_{n}\left(K+K^{\prime}\right) \geq \mathrm{r}_{n}(K)+\mathrm{r}_{n}\left(K^{\prime}\right), \\
& 2^{(p-1) / p} \mathrm{r}_{i}\left(K+{ }_{p} K^{\prime}\right) \geq \mathrm{r}_{i}\left(K+K^{\prime}\right) \geq \frac{1}{\sqrt{2}}\left(\mathrm{r}_{i}(K)+\mathrm{r}_{i}\left(K^{\prime}\right)\right),
\end{aligned}
$$

$i=1, \ldots, n-1$, which shows inequalities (7) and (8). Again, since $K, K^{\prime} \subseteq$ $K+{ }_{p} K^{\prime}$, then

$$
\mathrm{r}_{i}\left(K+{ }_{p} K^{\prime}\right) \geq \max \left\{\mathrm{r}_{i}(K), \mathrm{r}_{i}\left(K^{\prime}\right)\right\}
$$

which, together with the fact that $\max \{a, b\} \geq 1 / 2^{(3 p-2) /(2 p)}(a+b)$ for $p \geq 2$ and $a, b \geq 0$, leads to inequality (9).

So, we have to show that these inequalities are best possible. For the first one, with $K=K^{\prime}$ we get

$$
2^{(p-1) / p} \mathrm{r}_{n}\left(K+{ }_{p} K\right)=2^{(p-1) / p} 2^{1 / p} \mathrm{r}_{n}(K)=\mathrm{r}_{n}(K)+\mathrm{r}_{n}(K) .
$$

Next we fix $i \in\{1, \ldots, n-1\}$ and consider the $i$-dimensional unit balls $B_{i, L}$ and $B_{i, L^{\prime}}$ of the following $i$-dimensional linear subspaces: if $2 i>n$, let $L=\operatorname{lin}\left\{\mathrm{e}_{1}, \ldots, \mathrm{e}_{2 i-n}, \mathrm{e}_{2 i-n+1}, \ldots, \mathrm{e}_{i}\right\}, L^{\prime}=\operatorname{lin}\left\{\mathrm{e}_{1}, \ldots, \mathrm{e}_{2 i-n}, \mathrm{e}_{i+1}, \ldots, \mathrm{e}_{n}\right\} ;$ 
if $2 i \leq n$, let

$$
L=\operatorname{lin}\left\{\mathrm{e}_{1}, \ldots, \mathrm{e}_{i}\right\}, \quad L^{\prime}=\operatorname{lin}\left\{\mathrm{e}_{i+1}, \ldots, \mathrm{e}_{2 i}\right\} .
$$

Clearly, $\mathrm{r}_{i}\left(B_{i, L}\right)=\mathrm{r}_{i}\left(B_{i, L^{\prime}}\right)=1$. We notice also that, since $B_{i, L}, B_{i, L^{\prime}}$ are 0 -symmetric, then $B_{i, L}+{ }_{p} B_{i, L^{\prime}}$ is also 0-symmetric, and then

$$
\mathrm{r}_{i}\left(B_{i, L}+{ }_{p} B_{i, L^{\prime}}\right)=\max _{\bar{L} \in \mathcal{L}_{i}^{n}} \mathrm{r}\left(\left(B_{i, L}+{ }_{p} B_{i, L^{\prime}}\right) \cap \bar{L} ; \bar{L}\right) .
$$

Let $1 \leq p \leq 2$. We are going to show that

$$
\mathrm{r}\left(\left(B_{i, L}+{ }_{p} B_{i, L^{\prime}}\right) \cap \bar{L} ; \bar{L}\right) \leq 2^{(2-p) /(2 p)}
$$

for all $\bar{L} \in \mathcal{L}_{i}^{n}$, which will imply that

$$
\begin{aligned}
2^{(2-p) /(2 p)}=\frac{1}{2^{(3 p-2) /(2 p)}}(1+1) & =\frac{1}{2^{(3 p-2) /(2 p)}}\left(\mathrm{r}_{i}\left(B_{i, L}\right)+\mathrm{r}_{i}\left(B_{i, L^{\prime}}\right)\right) \\
& \leq \mathrm{r}_{i}\left(B_{i, L}+{ }_{p} B_{i, L^{\prime}}\right) \leq 2^{\frac{2-p}{2 p}},
\end{aligned}
$$

i.e., $\mathrm{r}_{i}\left(B_{i, L}+{ }_{p} B_{i, L^{\prime}}\right)=1 / 2^{(3 p-2) /(2 p)}\left(\mathrm{r}_{i}\left(B_{i, L}\right)+\mathrm{r}_{i}\left(B_{i, L^{\prime}}\right)\right)$, as required.

Let $L^{\prime \prime}=\operatorname{lin}\left\{\mathrm{e}_{j+1}, \ldots, \mathrm{e}_{n}\right\}$, where $j=2 i-n$ if $2 i>n$ and $j=0$ if $2 i \leq n$. In both cases,

$$
\begin{aligned}
\operatorname{dim}\left(\bar{L} \cap L^{\prime \prime}\right) & =\operatorname{dim} \bar{L}+\operatorname{dim} L^{\prime \prime}-\operatorname{dim}\left(\bar{L}+L^{\prime \prime}\right)=i+n-j-\operatorname{dim}\left(\bar{L}+L^{\prime \prime}\right) \\
& \geq i+n-j-n=i-j \geq 1 .
\end{aligned}
$$

Therefore, since $\operatorname{dim}\left(B_{i, L}+{ }_{p} B_{i, L^{\prime}}\right)=n$, then, for all $\bar{L} \in \mathcal{L}_{i}^{n}$, we can always find $z \in \operatorname{relbd}\left(B_{i, L}+{ }_{p} B_{i, L^{\prime}}\right) \cap \bar{L} \cap L^{\prime \prime}, z \neq 0$. Moreover, notice that, in particular, $z \in \operatorname{lin}\left\{\mathrm{e}_{j+1}, \ldots, \mathrm{e}_{2 i-j}\right\}$, and so it can be expressed in the form $z=x+x^{\prime} \in \operatorname{lin}\left\{\mathrm{e}_{j+1}, \ldots, \mathrm{e}_{i}\right\}+\operatorname{lin}\left\{\mathrm{e}_{i+1}, \ldots, \mathrm{e}_{2 i-j}\right\}=\left(L \cap L^{\prime \prime}\right)+\left(L^{\prime} \cap L^{\prime \prime}\right) ;$ observe that $x, x^{\prime}$ lie in orthogonal subspaces. Writing $u=z /|z|_{2}$, we have

$$
|z|_{2}=\langle z, u\rangle \leq h\left(B_{i, L}+{ }_{p} B_{i, L^{\prime}}, u\right)=\left(h\left(B_{i, L}, u\right)^{p}+h\left(B_{i, L^{\prime}}, u\right)^{p}\right)^{1 / p}
$$

and since

$$
\begin{aligned}
h\left(B_{i, L}, u\right) & =\sup _{y \in B_{i, L}}\langle y, u\rangle=\frac{1}{|z|_{2}} \sup _{y \in B_{i, L}}\langle y, x\rangle=\frac{1}{|z|_{2}}\left\langle\frac{x}{|x|_{2}}, x\right\rangle \\
& =h\left(\left[-\frac{x}{|x|_{2}}, \frac{x}{|x|_{2}}\right], u\right)
\end{aligned}
$$

and analogously $h\left(B_{i, L^{\prime}}, u\right)=h\left(\left[-x^{\prime} /\left|x^{\prime}\right|_{2}, x^{\prime} /\left|x^{\prime}\right|_{2}\right], u\right)$, we obtain that

$$
\begin{aligned}
|z|_{2} & \leq\left(h\left(\left[-\frac{x}{|x|_{2}}, \frac{x}{|x|_{2}}\right], u\right)^{p}+h\left(\left[-\frac{x^{\prime}}{\left|x^{\prime}\right|_{2}}, \frac{x^{\prime}}{\left|x^{\prime}\right|_{2}}\right], u\right)^{p}\right)^{1 / p} \\
& =h\left(\left[-\frac{x}{|x|_{2}}, \frac{x}{|x|_{2}}\right]+p\left[-\frac{x^{\prime}}{\left|x^{\prime}\right|_{2}}, \frac{x^{\prime}}{\left|x^{\prime}\right|_{2}}\right], u\right)=h\left(B_{2, \operatorname{lin}\left\{x, x^{\prime}\right\}}^{p /(p-1)}, u\right) \\
& \leq \mathrm{R}\left(B_{2, \operatorname{lin}\left\{x, x^{\prime}\right\}}^{p /(p-1)} \leq 2^{(2-p) /(2 p)} \mathrm{R}\left(B_{2, \operatorname{lin}\left\{x, x^{\prime}\right\}}\right)=2^{(2-p) /(2 p)}\right.
\end{aligned}
$$


by (10). This implies $\mathrm{r}\left(\left(B_{i, L}+{ }_{p} B_{i, L^{\prime}}\right) \cap \bar{L} ; \bar{L}\right) \leq 2^{(2-p) /(2 p)}$, showing (13) and concluding the proof of the case $1 \leq p \leq 2$.

Now let $p \geq 2$. Notice that $\mathrm{r}_{i}\left(B_{i, L}+{ }_{p} B_{i, L^{\prime}}\right) \geq \mathrm{r}_{i}\left(B_{i, L}\right)=\mathrm{r}_{i}\left(B_{i, L^{\prime}}\right)=1$. So, it suffices to show that $\mathrm{r}\left(\left(B_{i, L}+{ }_{p} B_{i, L^{\prime}}\right) \cap \bar{L} ; \bar{L}\right) \leq 1$ for all $\bar{L} \in \mathcal{L}_{i}^{n}$. With an analogous argument as before, but using (10) when $p \geq 2$, we get that there exists $z \in \operatorname{relbd}\left(B_{i, L}+{ }_{p} B_{i, L^{\prime}}\right) \cap \bar{L} \cap L^{\prime \prime}$ such that

$$
|z|_{2} \leq \mathrm{R}\left(B_{2, \operatorname{lin}\left\{x, x^{\prime}\right\}}^{p /(p-1)}\right) \leq \mathrm{R}\left(B_{2, \operatorname{lin}\left\{x, x^{\prime}\right\}}\right)=1 .
$$

It shows that $\mathrm{r}\left(\left(B_{i, L}+{ }_{p} B_{i, L^{\prime}}\right) \cap \bar{L} ; \bar{L}\right) \leq 1$ and concludes the proof.

We deal again with the possible existence of a reverse inequality, showing Proposition 1.2 .

Proof of Proposition 1.2. In the case of the diameter we easily get, using (11) and (3), that

$$
\mathrm{r}_{1}\left(K+{ }_{p} K^{\prime}\right) \leq \mathrm{r}_{1}\left(K+K^{\prime}\right) \leq \mathrm{r}_{1}(K)+\mathrm{r}_{1}\left(K^{\prime}\right) .
$$

The inequality is tight, since the diameter is a continuous functional and equality is attained if $K^{\prime}=\{0\}$.

In order to show the non-existence of a reverse inequality, $i=2, \ldots, n$, we take the convex bodies

$$
K=\left[-\mathrm{e}_{1}, \mathrm{e}_{1}\right] \quad \text { and } \quad K^{\prime}=\sum_{k=2}^{i}\left[-\mathrm{e}_{k}, \mathrm{e}_{k}\right]
$$

Clearly $\mathrm{r}_{i}(K)=\mathrm{r}_{i}\left(K^{\prime}\right)=0$, because they have dimensions $\operatorname{dim} K=1$ and $\operatorname{dim} K^{\prime}=i-1$. However,

$\mathrm{r}_{i}\left(K+{ }_{p} K^{\prime}\right) \geq 2^{-(p-1) / p} \mathrm{r}_{i}\left(K+K^{\prime}\right)=2^{-(p-1) / p} \mathrm{r}_{i}\left(\sum_{k=1}^{i}\left[-\mathrm{e}_{k}, \mathrm{e}_{k}\right]\right)=2^{-(p-1) / p}$,

which shows the result.

\section{ON THE $p$-DIFFERENCE BODY OF A CONVEX SET}

The difference body of a convex body $K \in \mathcal{K}^{n}$ is defined as the Minkowski addition $K-K:=K+(-K)$ which, in particular, is a 0 -symmetric body. Thus, this operation defines the so called central symmetral of a convex body $K$, just taking $K^{0}=(K-K) / 2$ (see e.g. [4, p. 79] for properties of the central symmetrization).

Similarly, the $p$-difference body can be defined: for $K \in \mathcal{K}_{0}^{n}$, we take the $p$-sum $K-{ }_{p} K:=K+{ }_{p}(-K)$. We observe that $K-{ }_{p} K$ is also a 0 -symmetric convex body: in fact,

$$
\begin{aligned}
h\left(K-{ }_{p} K,-u\right)^{p} & =h(K,-u)^{p}+h(-K,-u)^{p}=h(-K, u)^{p}+h(K, u)^{p} \\
& =h\left(K-{ }_{p} K, u\right)^{p} .
\end{aligned}
$$

In [3], a sharp Rogers-Shephard inequality for the $p$-difference body of a planar convex body was obtained, i.e., the best (upper) bound for the volume 
of the set $K-{ }_{p} K$ in terms of the volume of the original body $K$. Here we are interested in obtaining upper and lower bounds for the in- and outer radii of the $p$-difference body $K-{ }_{p} K$ in terms of the ones of $K$. In [9, Proposition 4.2] we already studied the behavior of the radii regarding the usual difference body: for all $i=1, \ldots, n$,

$$
\begin{aligned}
\sqrt{2} \sqrt{\frac{i+1}{i}} \mathrm{R}_{i}(K) & \leq \mathrm{R}_{i}(K-K) \leq 2 \mathrm{R}_{i}(K), \\
2 \mathrm{r}_{i}(K) & \leq \mathrm{r}_{i}(K-K)<2(i+1) \mathrm{r}_{i}(K) .
\end{aligned}
$$

Next proposition extends the above results to the $p$-difference body, $p \geq 1$, showing moreover that the bounds in Theorems 1.1 and 1.2 can be improved and that, in this particular case, there are non-trivial reverse inequalities (cf. Propositions 1.1 and 1.2 .

Proposition 3.1. Let $K \in \mathcal{K}_{0}^{n}$. Then for all $i=1, \ldots, n$ and all $p \geq 1$,

$$
\begin{aligned}
& \mathrm{R}_{i}\left(K-{ }_{p} K\right) \leq 2 \mathrm{R}_{i}(K), \\
& 2^{1 / p-1 / 2} \sqrt{\frac{i+1}{i}} \mathrm{R}_{i}(K) \leq \mathrm{R}_{i}\left(K-{ }_{p} K\right) \quad \text { if } 1 \leq p \leq 2, \\
& \max \left\{2^{1 / p-1 / 2} \sqrt{\frac{i+1}{i}}, 1\right\} \mathrm{R}_{i}(K) \leq \mathrm{R}_{i}\left(K{ }_{-p} K\right) \quad \text { if } p \geq 2 \text {. } \\
& 2^{1 / p} \mathrm{r}_{i}(K) \leq \mathrm{r}_{i}\left(K-{ }_{p} K\right)<2(i+1) \mathrm{r}_{i}(K) .
\end{aligned}
$$

Inequalities (15), (16) and the lower bound in (18) are best possible.

Proof. By (11) we have, for all $i=1, \ldots, n$, that

$$
\frac{1}{2^{(p-1) / p}} \mathrm{R}_{i}(K-K) \leq \mathrm{R}_{i}\left(K-{ }_{p} K\right) \leq \mathrm{R}_{i}(K-K),
$$

and analogously for the inner radii. Then applying (14), together with the fact that $K \subseteq K-{ }_{p} K^{\prime}$, we directly get the required inequalities.

We observe that if $p \geq 2$, then $\max \left\{2^{1 / p-1 / 2} \sqrt{(i+1) / i}, 1\right\}=1$ for all $i \geq 2$ and all $p \geq(2 \log 2) / \log (2 i /(i+1)) \in(2,4.81 \ldots)$.

So we deal with the sharpness of the inequalities, starting with the left hand side in (18). In this case, just notice that if $K$ is a 0 -symmetric convex body then $K=-K$ and hence $\mathrm{r}_{i}\left(K-{ }_{p} K\right)=\mathrm{r}_{i}\left(K+{ }_{p} K\right)=2^{1 / p} \mathrm{r}_{i}(K)$.

Next we study (15). We fix $i \in\{1, \ldots, n\}$ and consider the convex body $K=\left[0, \mathrm{e}_{1}\right]+\sum_{j=i+1}^{n}\left[-\mathrm{e}_{j}, \mathrm{e}_{j}\right]$, for which it clearly holds

$$
\mathrm{R}_{i}(K)=\mathrm{R}\left(K \mid \operatorname{lin}\left\{\mathrm{e}_{1}, \ldots, \mathrm{e}_{i}\right\}\right)=\mathrm{R}\left(\left[0, \mathrm{e}_{1}\right]\right)=\frac{1}{2} ;
$$

here, if $i=n$ we are taking $K=\left[0, \mathrm{e}_{1}\right]$. Now, on the one hand we notice that $\left(K-{ }_{p} K\right) \mid \operatorname{lin}\left\{\mathrm{e}_{1}, \ldots, \mathrm{e}_{i}\right\}=\left[0, \mathrm{e}_{1}\right]+{ }_{p}\left[-\mathrm{e}_{1}, 0\right]$ and that, by (2),

$$
\left[-\mathrm{e}_{1}, \mathrm{e}_{1}\right]=\operatorname{conv}\left(\left[0, \mathrm{e}_{1}\right] \cup\left[-\mathrm{e}_{1}, 0\right]\right) \subseteq\left[0, \mathrm{e}_{1}\right]+{ }_{p}\left[-\mathrm{e}_{1}, 0\right] \subseteq\left[-\mathrm{e}_{1}, \mathrm{e}_{1}\right],
$$

i.e., $\left(K-{ }_{p} K\right) \mid \operatorname{lin}\left\{\mathrm{e}_{1}, \ldots, \mathrm{e}_{i}\right\}=\left[0, \mathrm{e}_{1}\right]+{ }_{p}\left[-\mathrm{e}_{1}, 0\right]=\left[-\mathrm{e}_{1}, \mathrm{e}_{1}\right]$. On the other hand we observe that $\operatorname{conv}(K \cup(-K))=C_{n-i+1}$ is the $(n-i+1)$-dimensional 
cube of edge-length 2 contained in $\operatorname{lin}\left\{\mathrm{e}_{1}, \mathrm{e}_{i+1}, \ldots, \mathrm{e}_{n}\right\}$ and thus, by (2), we get that for all $L \in \mathcal{L}_{i}^{n}$

$$
\begin{aligned}
\mathrm{R}\left(\left(K-{ }_{p} K\right) \mid L\right) & \geq \mathrm{R}(\operatorname{conv}(K \cup(-K)) \mid L)=\mathrm{R}\left(C_{n-i+1} \mid L\right) \\
& \geq \mathrm{R}\left(C_{n-i+1} \mid \operatorname{lin}\left\{\mathrm{e}_{1}, \ldots, \mathrm{e}_{i}\right\}\right)=\mathrm{R}\left(\left[-\mathrm{e}_{1}, \mathrm{e}_{1}\right]\right) \\
& =\mathrm{R}\left(\left(K-{ }_{p} K\right) \mid \operatorname{lin}\left\{\mathrm{e}_{1}, \ldots, \mathrm{e}_{i}\right\}\right) .
\end{aligned}
$$

Therefore,

$$
\mathrm{R}_{i}\left(K-{ }_{p} K\right)=\mathrm{R}\left(\left(K{ }_{p} K\right) \mid \operatorname{lin}\left\{\mathrm{e}_{1}, \ldots, \mathrm{e}_{i}\right\}\right)=1=2 \mathrm{R}_{i}(K) .
$$

Finally we consider the equality case in (17).

If $i=n$, let $S_{n}$ be the $n$-dimensional simplex, embedded in $\mathbb{R}^{n+1}$, lying in the hyperplane $\left\{x=\left(x_{1}, \ldots, x_{n+1}\right) \in \mathbb{R}^{n+1}: \sum_{j=1}^{n+1} x_{j}=0\right\}$, given by

$$
S_{n}=\operatorname{conv}\left\{p_{k}: p_{k k}=\frac{n}{n+1}, p_{k j}=\frac{-1}{n+1} \text { for } j \neq k, k=1, \ldots, n+1\right\} \text {. }
$$

Since $S_{n}-{ }_{p} S_{n}$ is a 0 -symmetric $n$-dimensional convex body, then

$$
\mathrm{R}_{n}\left(S_{n}{ }_{-} S_{n}\right)=\max \left\{h\left(S_{n}-_{p} S_{n}, u\right):|u|_{2}=1 \text { and } \sum_{j=1}^{n+1} u_{j}=0\right\} .
$$

Let $u \in \mathbb{R}^{n+1}$ with $|u|_{2}=1$ and $\sum_{j=1}^{n+1} u_{j}=0$. Recall that the value of the support function of a convex body at any vector is attained in an extreme point (cf. e.g. [12, Theorem 5.6]), so, in order to compute $h\left(S_{n}, u\right)$ it suffices to consider the vertices of $S_{n}$. Since

$$
\left\langle p_{k}, u\right\rangle=\frac{n}{n+1} u_{k}-\frac{1}{n+1} \sum_{j \neq k} u_{j}=u_{k},
$$

then $h\left(S_{n}, u\right)=\max \left\{\left\langle p_{k}, u\right\rangle: k=1, \ldots, n+1\right\}=\max \left\{u_{1}, \ldots, u_{n+1}\right\}$. Without loss of generality we may assume that $u_{1} \geq \cdots \geq u_{n+1}$. Hence

$$
\begin{aligned}
h\left(S_{n}-{ }_{p} S_{n}, u\right)^{p} & =h\left(S_{n}, u\right)^{p}+h\left(-S_{n}, u\right)^{p}=h\left(S_{n}, u\right)^{p}+h\left(S_{n},-u\right)^{p} \\
& =u_{1}^{p}+\left(-u_{n+1}\right)^{p} .
\end{aligned}
$$

We observe that $u_{1} \geq 0$ and $u_{n+1} \leq 0$. Then, by elementary calculations it can be shown that the maximum of the function $u_{1}^{p}+\left(-u_{n+1}\right)^{p}, 1 \leq p \leq 2$, under the conditions $|u|_{2}=1$ and $\sum_{j=1}^{n+1} u_{j}=0$, is attained in the point $(1 / \sqrt{2}, 0, \ldots, 0,-1 / \sqrt{2})$. Therefore,

$$
\mathrm{R}_{n}\left(S_{n}-{ }_{p} S_{n}\right)=\left(\frac{1}{2^{p / 2}}+\frac{1}{2^{p / 2}}\right)^{1 / p}=2^{1 / p-1 / 2} .
$$

Since $\mathrm{R}_{n}\left(S_{n}\right)=\sqrt{n /(n+1)}$, then we get the required equality:

$$
\mathrm{R}_{n}\left(S_{n}-{ }_{p} S_{n}\right)=2^{1 / p-1 / 2}=2^{1 / p-1 / 2} \sqrt{\frac{n+1}{n}} \mathrm{R}_{n}\left(S_{n}\right) .
$$


If $i<n$, we take the $i$-dimensional simplex $S_{i}$ and consider the convex body $K=S_{i}+M C_{n-i}$, where $C_{n-i} \subset\left(\text { aff } S_{i}\right)^{\perp}$ represents the $(n-i)$-dimensional unit cube and $M>0$ is sufficiently large such that $\mathrm{R}_{i}\left(K{ }_{-} K\right)=\mathrm{R}\left(S_{i}-{ }_{p} S_{i}\right)$ and $\mathrm{R}_{i}(K)=\mathrm{R}\left(S_{i}\right)$. The above argument gives the result.

Acknowledgement. The authors would like to thank E. Saorín, who called our attention to this problem, for enlightening discussions. We also would like to thank the anonymous referees for valuable comments and suggestions.

\section{REFERENCES}

[1] U. Betke, M. Henk, Estimating sizes of a convex body by successive diameters and widths, Mathematika 39 (2) (1992), 247-257.

[2] U. Betke, M. Henk, A generalization of Steinhagen's theorem, Abh. Math. Sem. Univ. Hamburg 63 (1993), 165-176.

[3] C. Bianchini, A. Colesanti, A sharp Rogers and Shephard inequality for the $p$ difference body of planar convex bodies, Proc. Amer. Math. Soc. 136 (7) (2008), $2575-2582$.

[4] T. Bonnesen and W. Fenchel, Theorie der konvexen Körper. Springer, Berlin, 1934, 1974. English translation: Theory of convex bodies. Edited by L. Boron, C. Christenson and B. Smith. BCS Associates, Moscow, ID, 1987.

[5] R. Brandenberg, Radii of regular polytopes, Discrete Comput. Geom. 33 (1) (2005), 43-55.

[6] S. Campi, P. Gronchi, Volume inequalities for $L_{p}$-zonotopes, Mathematika 53 (1) (2006), 71-80 (2007).

[7] Wm. J. Firey, p-means of convex bodies, Math. Scand. 10 (1962), 17-24.

[8] E. D. Gluskin, Norms of random matrices and diameters of finite-dimensional sets (in Russian), Mat. Sb. (N.S.) 120 (162) (1983), 180-189.

[9] B. González, M. A. Hernández Cifre, Successive radii and Minkowski addition, Monatsh. Math. 166 (2012), 395-409.

[10] Y. Gordon, M. Junge, Volume formulas in $L_{p}$-spaces, Positivity 1 (1) (1997), 7-43.

[11] P. Gritzmann, V. Klee, Inner and outer $j$-radii of convex bodies in finite-dimensional normed spaces, Discrete Comput. Geom. 7 (1992), 255-280.

[12] P. M. Gruber, Convex and Discrete Geometry. Springer, Berlin Heidelberg, 2007.

[13] M. Henk, A generalization of Jung's theorem, Geom. Dedicata 42 (1992), 235-240.

[14] M. Henk, M. A. Hernández Cifre, Intrinsic volumes and successive radii, J. Math. Anal. Appl. 343 (2) (2008), 733-742.

[15] M. Henk, M. A. Hernández Cifre, Successive minima and radii, Canad. Math. Bull. 52 (3) (2009), 380-387.

[16] E. Lutwak, The Brunn-Minkowski-Firey theory, I, J. Differential Geom. 38 (1) (1993), 131-150.

[17] E. Lutwak, The Brunn-Minkowski-Firey theory, II, Adv. Math. 118 (2) (1996), 244294.

[18] E. Lutwak, D. Yang, G. Zhang, On the $L_{p}$-Minkowski problem, Trans. Amer. Math. Soc. 356 (11) (2004), 4359-4370.

[19] G. Ya. Perel'man, On the $k$-radii of a convex body, (Russian) Sibirsk. Mat. Zh. 28 (4) (1987), 185-186. English translation: Siberian Math. J. 28 (4) (1987), 665-666.

[20] A. Pietsch, s-numbers of operators in Banach spaces, Studia Math. 51 (1974), 201223.

[21] A. Pietsch, Operator Ideals. VEB Deutscher Verlag der Wissenschaften, Berlin, 1978. 
[22] S. V. Puhov, Inequalities for the Kolmogorov and Bernšter̆n widths in Hilbert space, (Russian) Mat. Zametki 25 (4) (1979), 619-628, 637. English translation: Math. Notes 25 (4) (1979), 320-326.

[23] R. Schneider, Convex Bodies: The Brunn-Minkowski Theory, Cambridge University Press, Cambridge, 1993.

[24] S. B. Steckin, On the best approximation of given classes of functions by arbitrary polynomials (in Russian), Uspekhi Mat. Nauk 9 (1954), 133-134.

Departamento de Matemáticas, Universidad de Murcia, Campus de EspinarDO, 30100-MurCIA, SPAIN

E-mail address: bgmerino@um.es

E-mail address: mhcifre@um.es 